\title{
A Consistent Model of the Interstellar Gamma-Ray Emission to Interpret Fermi LAT Observations of Diffuse Emissions
}

\section{Elena Orlando ${ }^{1}$}

Kavli Institute for Particle Astrophysics and Cosmology and Hansen Experimental Physics Laboratory, Stanford University, CA (USA)

E-mail: orlandele@gmail.com

\begin{abstract}
Cosmic rays interacting with gas and photons in the Galaxy produce interstellar gamma-ray emission, which accounts for most of the photons detected at gamma-ray energies. Models of this interstellar gamma-ray emission have to be very accurate for interpreting the high-quality observations by Fermi Large Area Telescope (LAT). Standard models, used as reference models for analyses of the Fermi LAT data, show spatial discrepancies with respect to the data, underlining the necessity of more realistic models.

We show that cosmic-ray propagation models that account for observations of the interstellar synchrotron emission in radio and microwave produce spatial and spectral distributions of the large-scale inverse-Compton component of the interstellar gamma-ray emission significantly different from the ones usually used in studies of Fermi LAT data. For example, models based on synchrotron observations produce a more peaked inverse-Compton emission in the inner Galaxy with respect to the standard models used to analyze Fermi LAT data.
\end{abstract}

This paper discusses the results based on Orlando (2019) Physical Review D 99, 043007 and Orlando (2018) MNRAS 475, 2724. Details are reported in those papers.

The author acknowledges support from NASA Grants No. NNX16AF27G.

36th International Cosmic Ray Conference -ICRC2019-

July 24th - August 1st, 2019

Madison, WI, U.S.A.

\footnotetext{
${ }^{1}$ Speaker

(C) Copyright owned by the author(s) under the terms of the Creative Commons 
A Consistent Model of the Interstellar Gamma-Ray Emission

This paper discusses the results based on Orlando (2019) Physical Review D 99, 043007 and Orlando (2018) MNRAS 475, 2724. Details are reported in those papers. 